\title{
Efficacy and safety of general anesthesia combined with paravertebral blockade on postoperative recovery in patients undergoing pulmonary surgery: a systematic review and meta-analysis
}

\author{
Pusheng Ren ${ }^{1}$, Yu Du ${ }^{1}$, Guangquan $\mathrm{He}^{1}$, Dan Jiang ${ }^{2}$ \\ ${ }^{1}$ Department of Anesthesiology, Nanchong Central Hospital Affiliated North Sichuan Medical College, Nanchong, China; ${ }^{2}$ Department of \\ Neurosurgery, Affiliated Hospital of North Sichuan Medical College, Nanchong, China \\ Contributions: (I) Conception and design: P Ren, Y Du; (II) Administrative support: G He; (III) Provision of study materials or patients: P Ren, Y Du, \\ D Jiang; (IV) Collection and assembly of data: All authors; (V) Data analysis and interpretation: All authors; (VI) Manuscript writing: All authors; (VII) \\ Final approval of manuscript: All authors. \\ Correspondence to: Pusheng Ren. Department of Anesthesiology, Nanchong Central Hospital Affiliated North Sichuan Medical College, No. 97, \\ Renmin South Road, Nanchong, China. Email: jibuluo@163.com.
}

Background: Research on analgesic effect, stress response, and lung function of thoracic epidural blockade (TEB) and paravertebral blockade (PVB) are inconsistent. This study conducted a meta-analysis of related literature, aiming at comparing the clinical efficacy and safety of two analgesic methods, and providing scientific evidence-based basis for clinical choice of analgesic methods.

Methods: PubMed, Embase, MEDLINE, Science Direct, Cochrane Library, CNKI, China Biomedical Resources Database, Wanfang Database, VIP, and Foreign Medical Journal Full-Text Service were searched. Keywords were as follows: thoracic epidural block (TEB), paravertebral blockade (PVB), paravertebral catheterization, thoracotomy, and analgesia. Two professionals independently screened documents and extracted data, and used Cochrane System Evaluator Manual (version 5.1.0) to repeatedly assess the bias risk of the documents included in the study.

Results: A total of 9 articles were included. Of the 9 RCTs in the present study, 5 described the allocation concealment in detail, 9 described the correct random allocation method, and 1 did not use the blind method. The visual simulation scores of the PVB group and TEB group at 24 and 48 h were not statistically significant [mean difference (MD): $-0.17,95 \%$ confidence interval (CI): -0.43 to $0.08, \mathrm{P}=0.18 ; \mathrm{MD}: 0.21$, 95\% CI: -0.06 to $0.48, \mathrm{P}=0.13]$. The fixed-effects model was used to analyze the incidence of hypotension, nausea, vomiting, and urinary retention. The results showed that there was significant difference between the PVB group and TEB group [hypotension: relative risk (RR): 0.16, 95\% CI: 0.06-0.46, P=0.0006; nausea: RR: 0.40, 95\% CI: 0.25-0.66, $\mathrm{P}=0.0002$; vomiting: RR: 0.23 , 95\% CI: 0.06-0.87, P=0.03; urinary retention: RR: $0.36,95 \%$ CI: $0.15-0.87, \mathrm{P}=0.02]$.

Discussion: The meta-analysis confirmed that PVB has the same analgesic effect and postoperative pulmonary function as epidural blockade in open thoracotomy lung surgery. In addition, PVB can reduce the incidence of analgesia-related complications and postoperative chronic pain.

Keywords: General anesthesia; paravertebral blockade; epidural blockade; lung surgery; meta-analysis

Submitted Jan 10, 2022. Accepted for publication Feb 18, 2022.

doi: $10.21037 /$ jtd-22-103

View this article at: https://dx.doi.org/10.21037/jtd-22-103

(c) Journal of Thoracic Disease. All rights reserved. 


\section{Introduction}

Open thoracotomy lung surgery has one of the highest incidences of postoperative pain after lung surgery, which directly affects the patient's pulmonary respiratory function and reduces ventilation efficiency. It can lead to atelectasis, pulmonary infection, respiratory distress, hypercapnia, and other pulmonary complications, and sometimes even chronic pain after thoracotomy. Therefore, it is important to control postoperative pain (1-3). In recent years, thoracic epidural blockade (TEB) has been the most commonly used method for open thoracotomy lung surgery analgesia, and is recognized as the "gold standard" of open thoracotomy lung surgery analgesia (4). Ding et al. found that epidural blockade can significantly improve the postoperative stress response and reduce the incidence of postoperative complications (5). However, epidural blockade can be accompanied by nausea, vomiting, hypotension, urinary retention, and a series of adverse reactions, and even lead to dura and epidural abscess.

Traditional thoracic and pulmonary surgery anesthesia is most commonly used for inhalation anesthesia or general intravenous anesthesia, or a combination of both (6). Paravertebral blockade (PVB) refers to the injection of anesthetics into the spinal nerve roots on both sides of the vertebral body and out of the intervertebral foramen to block the paravertebral nerve to achieve analgesic effect. It has the advantages of simplicity, efficacy, and reliability. Continuous blockade with a paravertebral catheter can achieve long-term analgesic effect, which is suitable for postoperative analgesia of the unilateral lung (7). At present, general anesthesia combined with PVB is widely used in single-lung ventilation thoracic lung surgery (8).

There are a number of studies, both locally and internationally, on the application of epidural and PVB in thoracotomy (9). Compared with epidural blockade, PVB has higher specificity, lower probability of hypotension, and less postoperative nausea, vomiting, and urinary retention. Raveglia et al. [2014] (10) found that 2 patients in PVB group had heart failure, 1 patient had myocardial infarction and 1 patient in TEB group had heart failure after operation, but the original text did not mention the correlation with analgesic measures. However, at present, results are inconsistent in terms of analgesic effect, stress response, and pulmonary function recovery of patients with the 2 methods.

In the field of medical research, systematic evaluation and meta-analysis are one of the important methods for producing high-quality research evidence in evidence-based medicine, and are one of the most frequently used research tools in clinical medicine. Systematic evaluation and metaanalysis mainly collect unpublished and published clinical research results all over the world comprehensively and systematically, and explore the safety and effectiveness of a specific clinical problem. The principles and methods of strict evaluation of clinical epidemiology literature were used to conduct evaluation and analysis, and literature that met the quality standards was screened out. Qualitative and quantitative syntheses was carried out, and comprehensive and reliable conclusions were obtained (11). At the same time, with the updating of new clinical research, metaanalysis can provide the latest knowledge and information as the basis for decision-making.

In the present study, we analyzed randomized controlled trials (RCTs) of PVB and epidural blockade analgesia after unilateral open thoracotomy lung surgery for systematic evaluation and meta-analysis using the Cochrane system. The purpose of the study was to evaluate the clinical efficacy and safety of postoperative recovery in patients with 2 types of analgesia methods, and to provide scientific evidence for the clinical selection of analgesia methods.

We present the following article in accordance with the PRISMA reporting checklist (available at https://jtd. amegroups.com/article/view/10.21037/jtd-22-103/rc).

\section{Methods}

\section{Literature retrieval}

PubMed, Embase, MEDLINE, Science Direct, Cochrane Library, CNKI, China Biomedical Resources Database, Wanfang Database, VIP, and Foreign Medical Journal FullText Service were searched. RCTs of PVB and epidural blockade analgesia after unilateral open thoracotomy lung surgery were searched from database establishment until April 24, 2021. It was necessary to manually retrieve articles published in journals to avoid omissions.

The retrieval strategy was as follows: (I) keywords for research object retrieval in English were searched; these included the following: epidural blockade, PVB, paravertebral tube placement, thoracotomy, analgesia drainage. Keywords in Chinese included the following: epidural blockade, PVB, paravertebral catheterization, thoracotomy, and analgesia; (II) keywords for outcome indictors in English included the following: visual analog scale (VAS) score of rest and activity at 24 and $48 \mathrm{~h}$ after operation, use of remedial measures, incidence of complications related to analgesia (including nausea and 
vomiting, hypotension, and urinary retention), incidence of pulmonary complications, success rate of operation, changes of respiratory function after operation, hospitalization time, and incidence of chronic pain after thoracotomy. Keywords in Chinese included: the following: VAS score at 24 and $48 \mathrm{~h}$ after surgery, incidence of analgesia-related complications (including nausea and vomiting, hypotension, and urinary retention), changes in postoperative respiratory function, and incidence of chronic pain after thoracotomy.

Research object search terms and observation index search terms are searched in pairwise combination, and more related references are included.

\section{Standards for inclusion and exclusion of literature}

The inclusion criteria were as follows: (I) prospective RCTs limited to Chinese and English; (II) patients were patients undergoing thoracic lung surgery; (III) intervention measures in the experimental group were general anesthesia combined with PVB after unilateral thoracotomy; the control group was treated with epidural blockade after unilateral thoracotomy; and (IV) outcome indicators: VAS score at 24 and $48 \mathrm{~h}$ after operation, incidence of analgesia-related complications (including nausea and vomiting, hypotension, and urinary retention), changes in postoperative respiratory function, and incidence of chronic pain after thoracotomy.

The exclusion criteria were as follows: (I) non-prospective RCTs; (II) patients underwent emergency surgery; (III) cases, reviews, conferences, reviews, and other non-research literatures; (IV) analgesic methods of non-PVB and nonepidural blockade, and (V) repeated publication or unable to contact the authors to obtain the original data.

\section{Postoperative recovery outcome indicators}

The postoperative recovery outcome indicators were VAS score at 24 and $48 \mathrm{~h}$ after operation and changes in postoperative respiratory function; safety indicators: incidence of analgesia-related complications (including nausea and vomiting, hypotension, and urinary retention) and incidence of chronic pain after thoracotomy.

\section{Data extraction}

The two researchers in this meta-analysis independently screened the literature and extracted data using Microsoft Excel to assess the methodological quality of the included studies, followed by cross-checking. If there was any disagreement, it can be solved through discussion. The main extracted data included the following: (I) general information included in the study: published journals, published dates, topics, first authors, and countries; (II) basic information of the research object: sex, age, and number of cases; (III) methods of operation and followup time of intervention; (IV) extraction of outcome indicators: VAS score at 24 and $48 \mathrm{~h}$ after surgery, incidence of analgesia-related complications (including nausea and vomiting, hypotension, and urinary retention), changes in postoperative respiratory function, and incidence of chronic pain after thoracotomy.

\section{Bias risk assessment}

The bias risk assessment of the included literature was repeated by 2 professionals in strict accordance with the 5 evaluation criteria of RCTs mentioned below. If differences were encountered, they were solved through discussion. The methodological quality of included studies was assessed using the Cochrane Handbook of Systematic Reviews of Interventions and the risk of bias assessment tool for RCTs. Evaluation criteria were as follows: (I) random methods; (II) hiding allocation; (III) blind method; (IV) missing visits; and $(\mathrm{V})$ judging selective bias.

\section{Statistical analysis}

RevMan 5.3 software was used to draw the risk assessment map of bias, and the risk bias of included references was evaluated. Data after sorting and screening were input into the software to draw the corresponding chart. The mean difference (MD) and its 95\% confidence interval (CI) were used for the measurement data, and the relative risk (RR) and its $95 \% \mathrm{CI}$ were used for the count data. Heterogeneity among the studies was explored by $\mathrm{I}^{2}$ test. When $\mathrm{P}>0.10$ and $\mathrm{I}^{2}<50 \%$, the fixed-effects model was used for the metaanalysis of forest map, indicating that heterogeneity of the included studies was small. When $\mathrm{P}<0.10$ and $\mathrm{I}^{2}>50 \%$, the random-effects model was used for the meta-analysis of forest map, indicating that the heterogeneity of included studies was large. The impact of total merger effect was studied, excluding the larger impact of the study. The random-effects model and the fixed-effects model were used to compare the results. According to the consistency of the results, the reliability of the combined results was analyzed. Funnel plots were used to determine publication bias. 


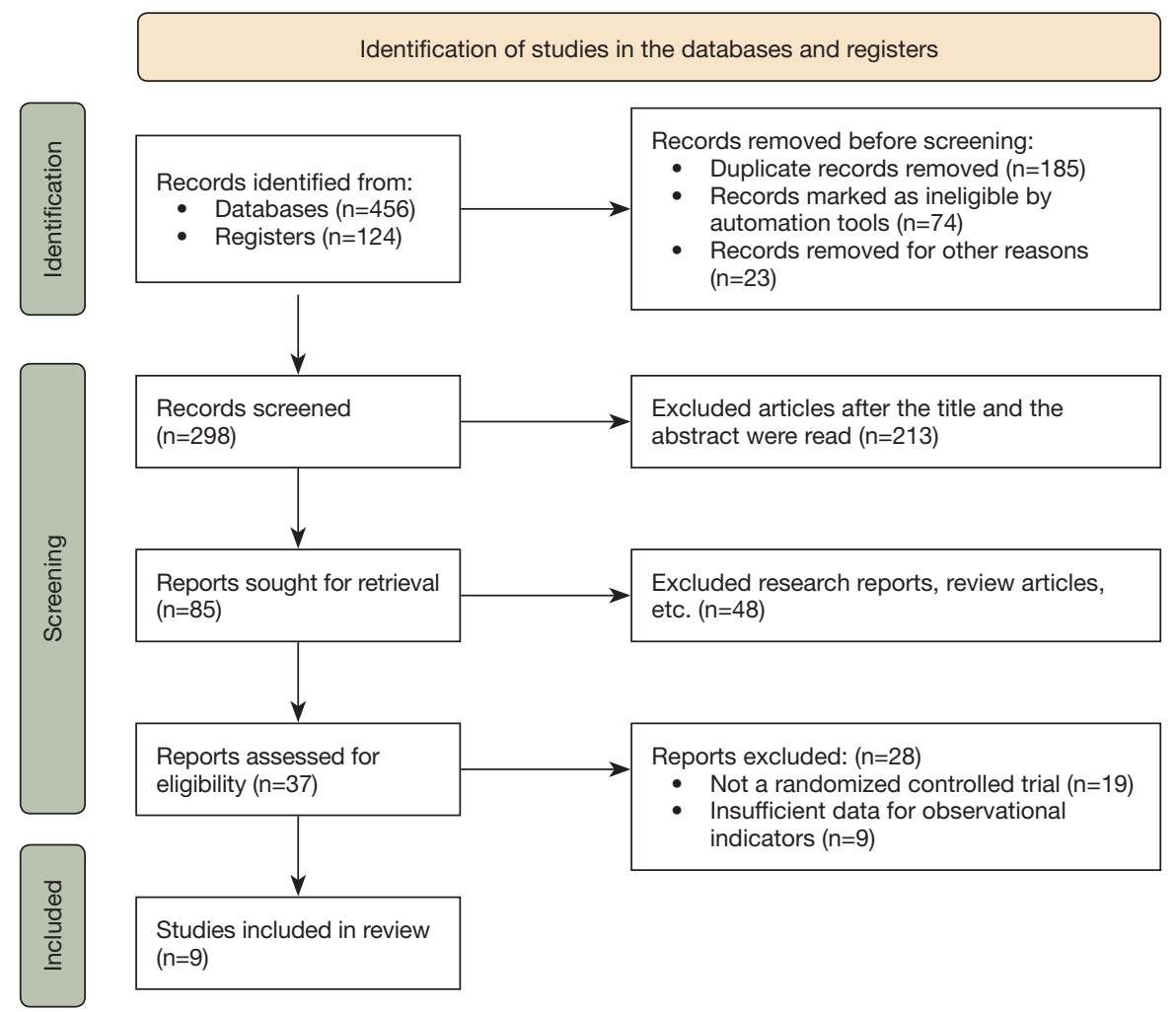

Figure 1 Flow chart of literature retrieval.

\section{Results}

\section{Search results and basic information of the literature}

A total of 456 articles were retrieved from the databases, and 124 articles were retrieved from the registers. In total, 185 repetitive articles, 74 unqualified articles, and 23 articles for other reasons were excluded. The remaining 298 articles were selected by title selection. After reading the abstracts, 213 articles were deleted and 85 articles remained. Fortyeight research reports and reviews were excluded, and the remaining 37 were included. After reading the full text, 19 non-RCTs were excluded, as relevant information could not be further extracted from the articles. Nine articles were excluded and 9 articles were finally included into the metaanalysis. The flowchart of literature retrieval is shown in Figure 1.

Nine articles met the inclusion criteria, with a total of 441 patients. In the 9 articles, the sample size ranged from 30 to 95 . The VAS scale at 24 and $48 \mathrm{~h}$ after surgery was described in detail in the references of all 9 articles and included the incidence of analgesia-related complications (including nausea and vomiting, hypotension, and urinary retention), changes in postoperative respiratory function, and incidence of chronic pain after thoracotomy. Table 1 lists the basic characteristics and scale score of the included literature.

\section{Risk bias evaluation results of references}

RevMan 5.3 software was used to draw the risk bias evaluation diagram and summary diagram of references (Figures 2,3). Of the 9 RCTs in the present study, 5 described the allocation concealment in detail, 9 described the correct random allocation method, and 1 did not use the blind method.

\section{Evaluation of postoperative recovery of patients}

\section{Meta-analysis results of VAS score}

In 5 references, the VAS scales at rest and their MDs at 24 and $48 \mathrm{~h}$ in the RCTs were analyzed. The VAS scores of the 5 articles were described by continuous variables.

Figures 4,5 show the 24 and $48 \mathrm{~h}$ VAS scales at rest in a forest map. Heterogeneity test of $24 \mathrm{~h}$ VAS scores 
Table 1 Basic characteristics and scale score of the included literature

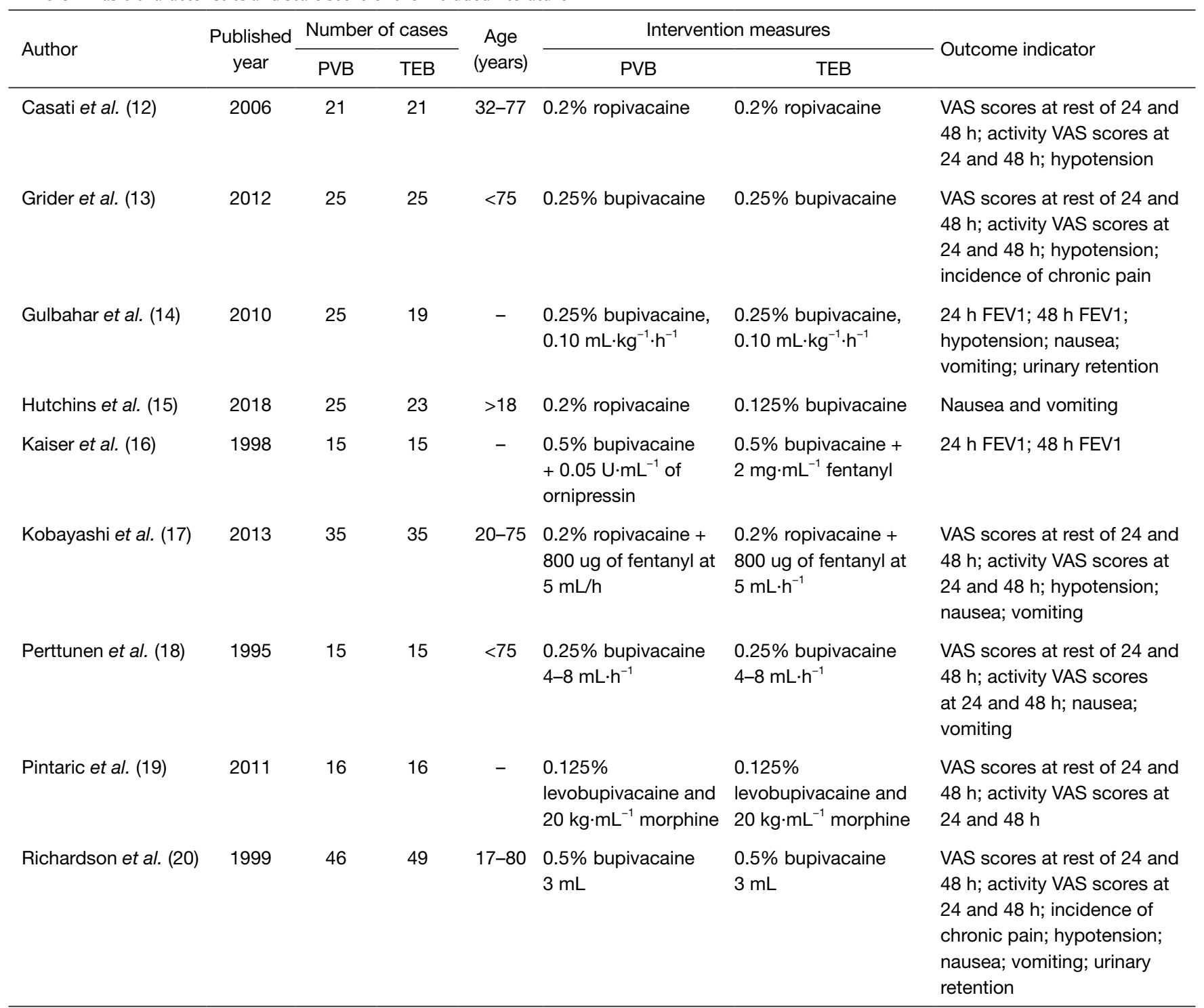

PVB, paravertebral blockade; TEB, thoracic epidural blockade; VAS, visual analog scale; FEV1, forced expiratory volume in $1 \mathrm{~s}$.

was performed, and the fixed-effects model was used to demonstrate that there was no statistically significant difference between the PVB group and TEB group (MD: $0.10,95 \%$ CI: -0.13 to $0.33, \mathrm{P}=0.39)$. Heterogeneity test of $48 \mathrm{~h}$ VAS scores was performed, and the randomeffects model was used to demonstrate that there was no statistically significant difference between the PVB group and TEB group (MD: $-0.07,95 \%$ CI: -0.53 to 0.39 , $\mathrm{P}=0.75)$.

Figures 6,7 show forest maps of VAS scores at 24 and $48 \mathrm{~h}$. Heterogeneity test of VAS scores at $24 \mathrm{~h}$ was performed, and the fixed-effects model was used to demonstrate there was no statistically significant difference between the PVB group and TEB group (MD: $-0.17,95 \%$ CI: -0.43 to $0.08, \mathrm{P}=0.18$ ). Heterogeneity test of VAS scores at $48 \mathrm{~h}$ was performed, and the fixed-effects model was used to demonstrate that there was no statistically significant difference between the PVB group and TEB group (MD: 0.21, 95\% CI: -0.06 to 0.48 , $\mathrm{P}=0.13)$.

Figure 8 is a funnel diagram of publication bias. The circles representing the research are distributed near the midline and are symmetrically distributed as a whole, 


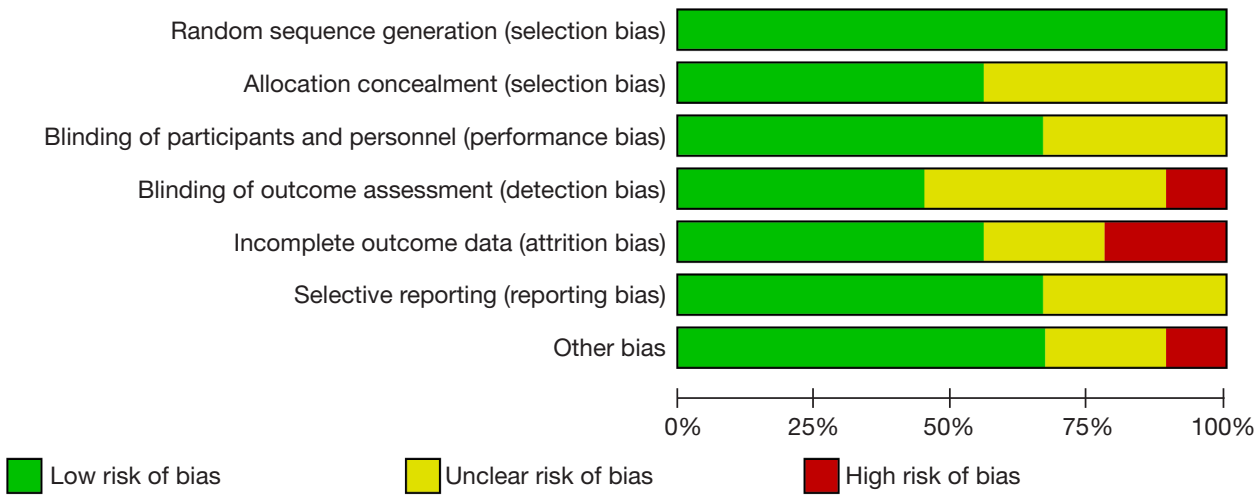

Figure 2 Risk bias evaluation chart of included articles.

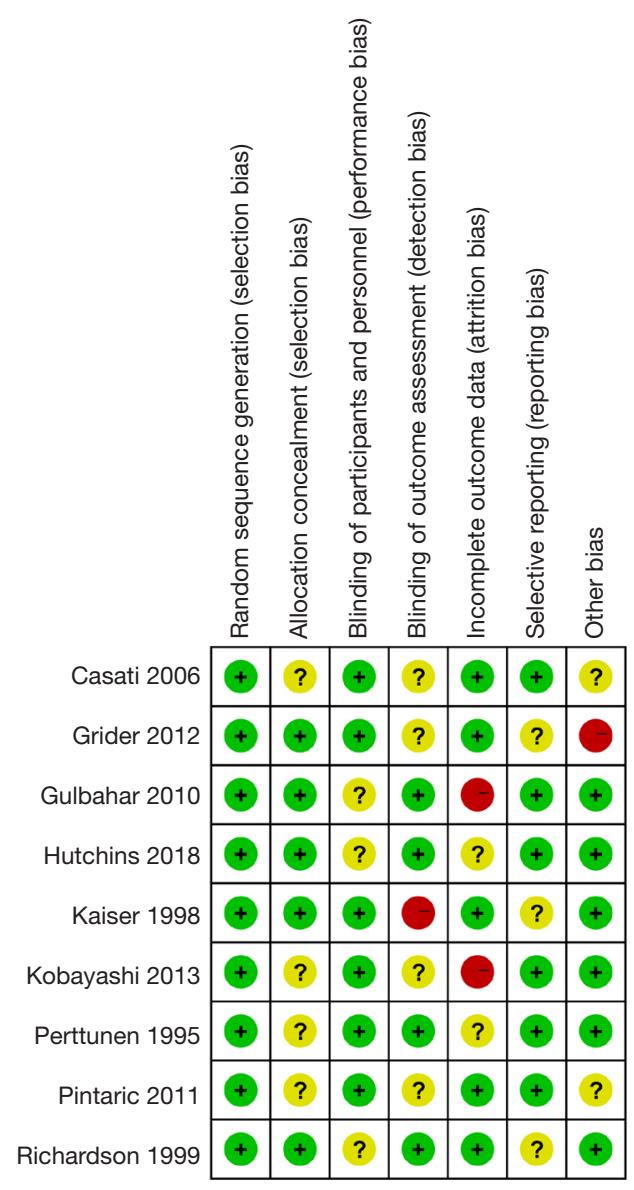

Figure 3 Summary of risk bias assessment for included literature. “+”, low of risk; “-”, high of risk; “?”, unclear. indicating that the included research has high accuracy and there is no publication bias.

\section{Meta-analysis results of respiratory function}

In 2 references $(14,16)$, the percentage of forced expiratory volume in $1 \mathrm{~s}(\mathrm{FEV} 1)$ at 24 and $48 \mathrm{~h}$ after operation at the preoperative level in the RCTs was analyzed.

The percentage heterogeneity test results of $24 \mathrm{~h} 1$-s maximum expiratory volume showed that $\chi^{2}=0.24, \mathrm{df}=1$, $\mathrm{I}^{2}=0 \%<50 \%$, and $\mathrm{P}=0.62$ (Figure 9), and there was no heterogeneity in each study group. The overall analysis using the fixed-effects model showed that there was no statistical significance between the PVB group and the TEB group (MD: 2.82, 95\% CI: -10.05 to $15.69, \mathrm{P}=0.67$ ).

The 48-h maximum expiratory volume in 1-s preoperative level percentage heterogeneity test results showed that $\chi^{2}=1.87, \mathrm{df}=1, \mathrm{I}^{2}=47 \%<50 \%$, and $\mathrm{P}=0.17$ (Figure 10); heterogeneity did not exist in the study group. The overall analysis using the fixed-effects model showed that there was no statistical significance between the PVB group and the TEB group (MD: 2.88, 95\% CI: -9.99 to $15.75, \mathrm{P}=0.66$ ).

\section{Meta-analysis results of the incidence of chronic pain after thoracotomy}

In 2 articles, the incidence of chronic pain after thoracotomy in RCTs was analyzed. Figure 11 shows a forest map of the fixed-effects model of the incidence of chronic pain after thoracotomy. Two studies used binary 


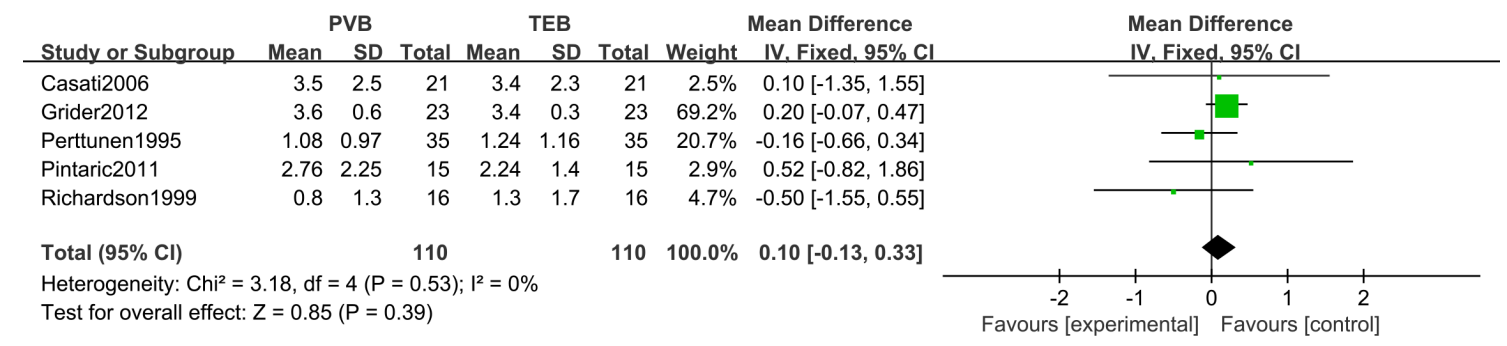

Figure 4 Forest map of visual analog sore scales at rest fixed-effects model of $24 \mathrm{~h}$. PVB, paravertebral blockade; TEB, thoracic epidural blockade; df, degree of freedom; SD, standard deviation; CI, confidence interval.

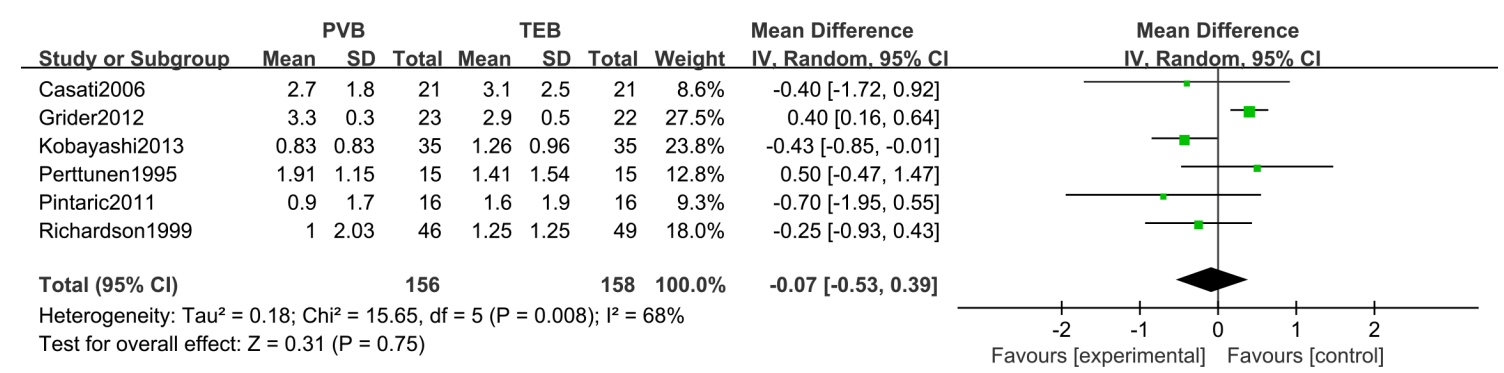

Figure 5 Forest map of visual analog score scales at rest random-effects model of 48 h. PVB, paravertebral blockade; TEB, thoracic epidural blockade; df, degree of freedom; SD, standard deviation; CI, confidence interval.

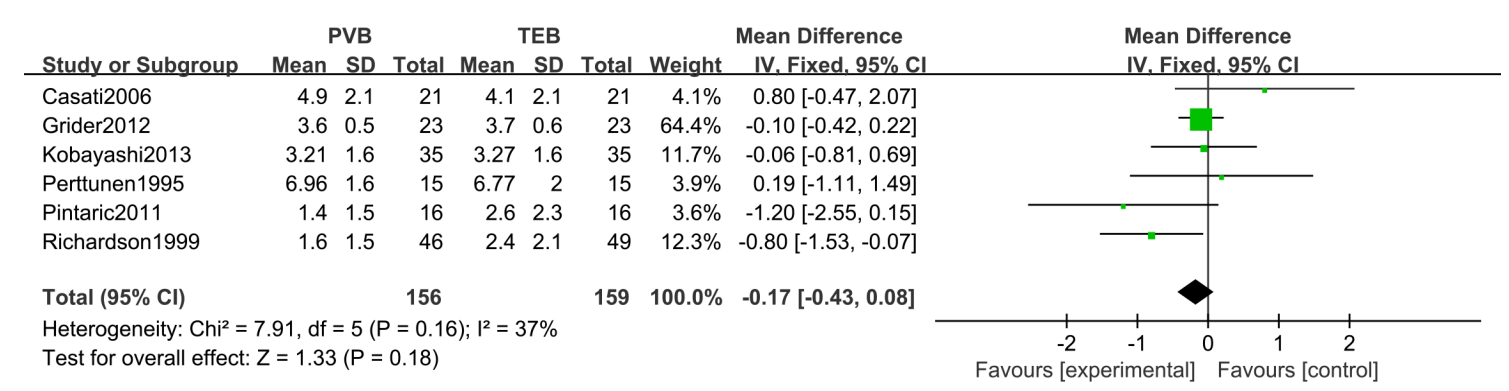

Figure 6 Forest map of activity visual analog scale scores fixed-effects model of 24 h. PVB, paravertebral blockade; TEB, thoracic epidural blockade; SD, standard deviation; df, degree of freedom; CI, confidence interval.

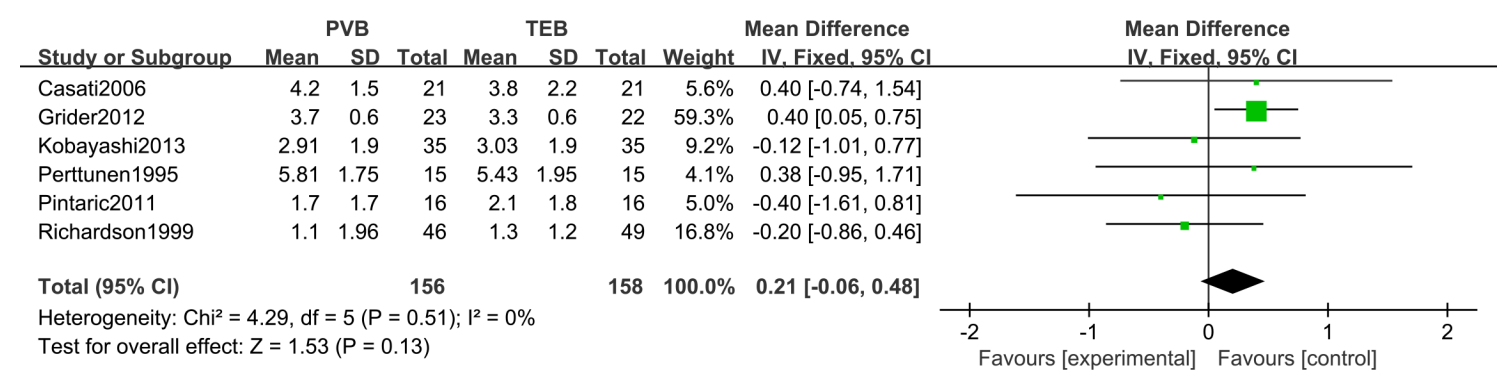

Figure 7 Forest map of activity visual analog scale scores fixed-effects model of 48 h. PVB, paravertebral blockade; TEB, thoracic epidural blockade; df, degree of freedom; SD, standard deviation; CI, confidence interval. 


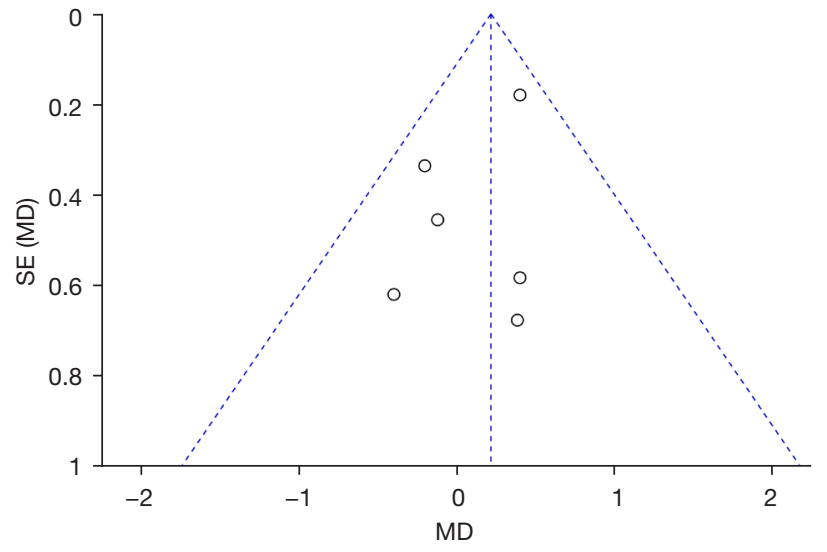

Figure 8 Funnel chart of bias. SE, standard error; MD, mean difference.

classification variables to describe the incidence of chronic pain after thoracotomy. There were 141 cases in total; 69 cases in the PVB group and 72 cases in the TEB group. Overall heterogeneity test was performed $\left(\chi^{2}=0.48, \mathrm{df}=1\right.$, $\left.\mathrm{I}^{2}=0 \%<50 \%, \mathrm{P}=0.49\right)$. There was no heterogeneity in each study group. The overall analysis using the fixed-effects model showed that the difference between the PVB group and the TEB group was not statistically significant (RR: 0.40, 95\% CI: $0.15-1.07, \mathrm{P}=0.07)$.

\section{Meta-analysis results of analgesia-related complications}

Five articles analyzed the incidence of hypotension, nausea, vomiting, and urinary retention in RCTs. Figures 12-15 are forest maps of the fixed-effects models of hypotension, nausea, vomiting, and urinary retention, respectively. The incidence of hypotension, nausea, vomiting, and urinary retention was described by 2 classification variables in 5 articles. There were 297 cases in total; 150 cases in the experimental group and 147 cases in the control group.

Overall heterogeneity was tested (hypotension $\chi^{2}=1.75, \mathrm{df}=4, \mathrm{I}^{2}=0 \%<50 \%, \mathrm{P}=0.78$; nausea $\chi^{2}=4.60, \mathrm{df}=4$, $\mathrm{I}^{2}=13 \%<50 \%, \mathrm{P}=0.33$; vomiting $\chi^{2}=0.38, \mathrm{df}=1, \mathrm{I}^{2}=0 \%<50 \%$, $\mathrm{P}=0.54$; urinary retention $\chi^{2}=1.34, \mathrm{df}=1, \mathrm{I}^{2}=25 \%<50 \%$, $\mathrm{P}=0.25$ ), and there was no heterogeneity among the studies. The overall analysis using the fixed-effects model shows that the difference between the experimental group and the control group was statistically significant (hypotension: RR: 0.16, 95\% CI: 0.06-0.46, $\mathrm{P}=0.0006$; nausea: RR: 0.40, 95\% CI: $0.25-0.66, \mathrm{P}=0.0002$; vomiting: $\mathrm{RR}: 0.23,95 \%$ CI: $0.06-0.87, P=0.03$; urinary retention: $R R: 0.36,95 \%$ CI: $0.15-0.87, \mathrm{P}=0.02)$. Figure 16 shows a funnel plot of analgesia-related complications. Circles were concentrated near the midline and were generally symmetrical, indicating no publication bias.

\section{Discussion}

Compared with epidural blockade, PVB has higher specificity. In the present study, the VAS scales at 24 and $48 \mathrm{~h}$ after treatment of the 2 schemes were analyzed. Metaresults showed that there was no significant difference in the analgesic effect between the 2 schemes (21). The VAS scores of PVB at $48 \mathrm{~h}$ after operation was lower than that of epidural blockade, showing good analgesic effect $(6,22)$.

Among the 9 RCTs in the present study, 5 described the allocation concealment in detail, 3 described the correct random allocation method, and 1 did not use the blind method. The most common complications related to analgesia included hypotension, nausea, vomiting, and urinary retention. The meta-results of the present study showed that the incidence of PVB group was significantly lower than that of TEB group (hypotension: RR: 0.16, 95\% CI: 0.06-0.46), $\mathrm{P}=0.0006$; nausea: RR: $0.40,95 \% \mathrm{CI}$ : $0.25-$ $0.66, \mathrm{P}=0.0002$; vomiting: $\mathrm{RR}: 0.23,95 \% \mathrm{CI}: 0.06-0.87$, $\mathrm{P}=0.03$; urinary retention: $\mathrm{RR}: 0.36,95 \% \mathrm{CI}: 0.15-0.87$, $\mathrm{P}=0.02$ ), reflecting the obvious advantage of $\mathrm{PVB}$, which is similar to the research results of Neuburger et al. (23).

For postoperative pulmonary function, a meta-analysis of FEV1 at 24 and $48 \mathrm{~h}$ after operation was conducted. The results showed that there was no significant difference between the PVB and TEB groups (24-h FEV1: MD: 2.82, 95\% CI: -10.05 to $15.69, \mathrm{P}=0.67$; 48-h FEV1: MD: 2.88 , 95\% CI: -9.99 to $15.75, \mathrm{P}=0.66)$. This is inconsistent with the research results of Koyyalamudi et al. (24). The reason could be that our meta-analysis does not include cardiac surgery, but only thoracic unilateral pulmonary resection. Surgical trauma and cardiopulmonary bypass will have a certain impact on respiratory function, and could be associated with different pulmonary function evaluation indicators (25). There was no significant difference in the effects of the 2 analgesic methods on postoperative pulmonary function; the sample size needs to be increased at a later stage.

During general anesthesia induction, due to the stimulation of intubation, it is easy to cause the body to release a large number of sympathetic excitement and vasoactive substances, resulting in stress reactions, such as increased blood pressure and heart rate. After extubation, shallow anesthesia, restlessness, wound pain, and tracheal 


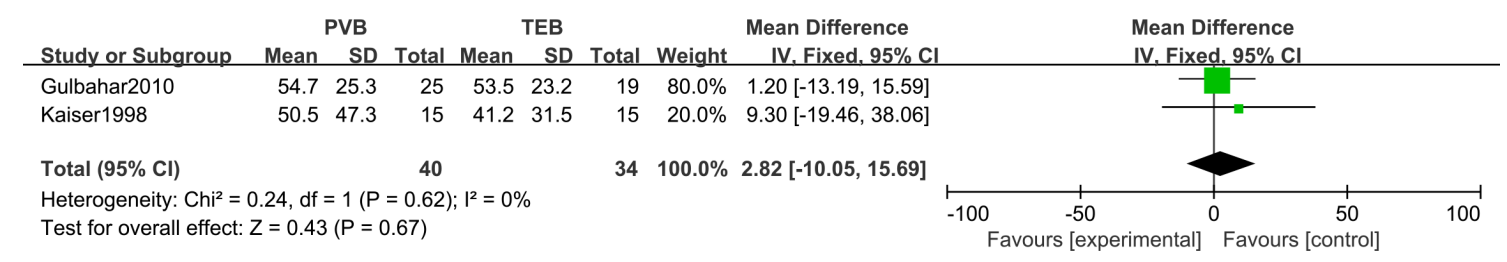

Figure 9 Forest map of the preoperative level percentage fixed-effects model of 24-h maximum expiratory volume in $1 \mathrm{~s}$. PVB, paravertebral blockade; TEB, thoracic epidural blockade; df, degree of freedom; SD, standard deviation; CI, confidence interval.

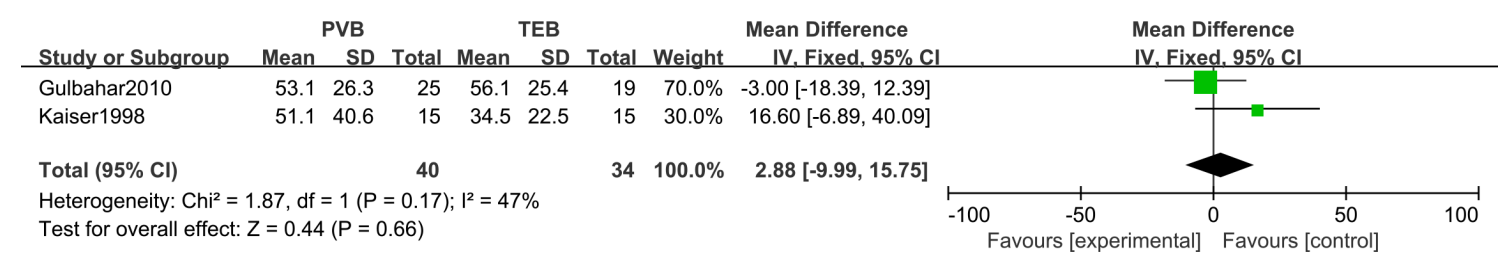

Figure 10 Forest map of the preoperative level percentage fixed-effects model of 48-h maximum expiratory volume in 1 s. PVB, paravertebral blockade; TEB, thoracic epidural blockade; df, degree of freedom; SD, standard deviation; CI, confidence interval.

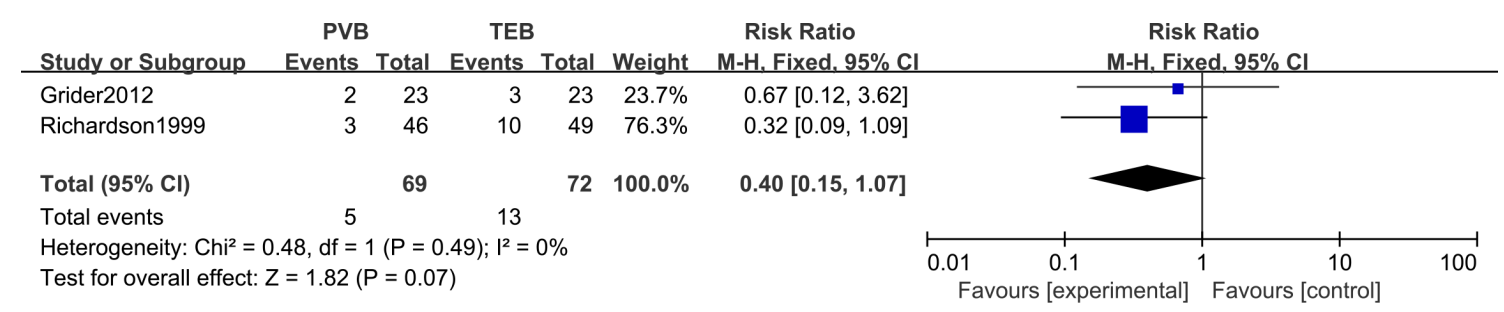

Figure 11 Forest map of the fixed-effects model of incidence of chronic pain after thoracotomy. PVB, paravertebral blockade; TEB, thoracic epidural blockade; df, degree of freedom; CI, confidence interval.

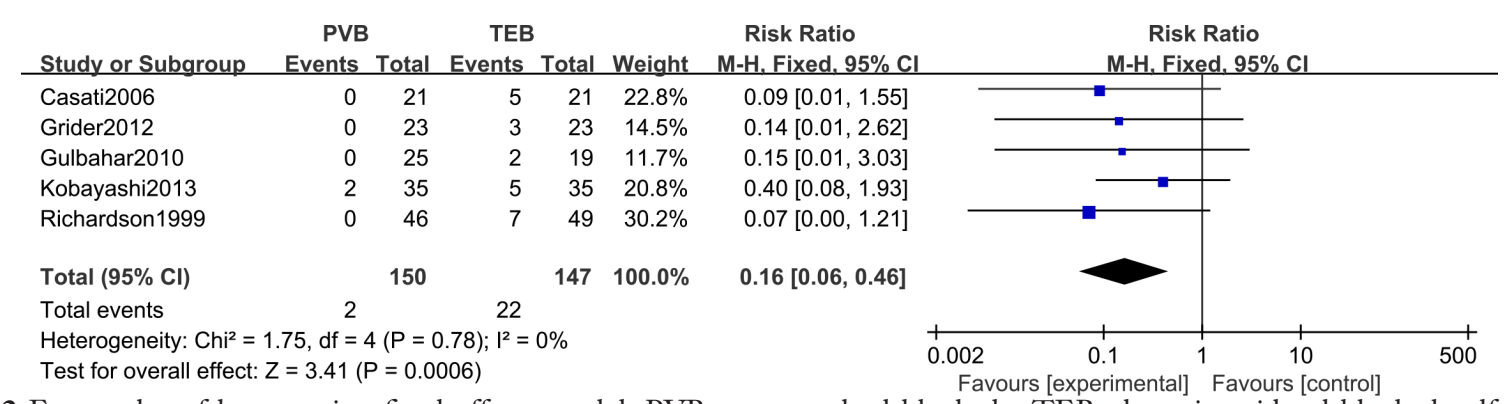

Figure 12 Forest plot of hypotension fixed-effects model. PVB, paravertebral blockade; TEB, thoracic epidural blockade; df, degree of freedom; CI, confidence interval.

stimulation of the respiratory tract could lead to a strong cardiovascular response during perioperative extubation, such as increased heart rate and elevated blood pressure (26,27). General anesthesia combined with PVB anesthesia significantly reduces the dosage of total anesthetics. Visoiu et al. (28) shows that PVB anesthesia was convenient, the anesthesia process was peaceful, and the postoperative recovery was faster. The extubation time was advanced, and the incidence of complications was low. PVB catheterization includes surgical catheterization and percutaneous puncture, and surgical catheterization can be caused by surgical operation. Percutaneous catheterization is more 


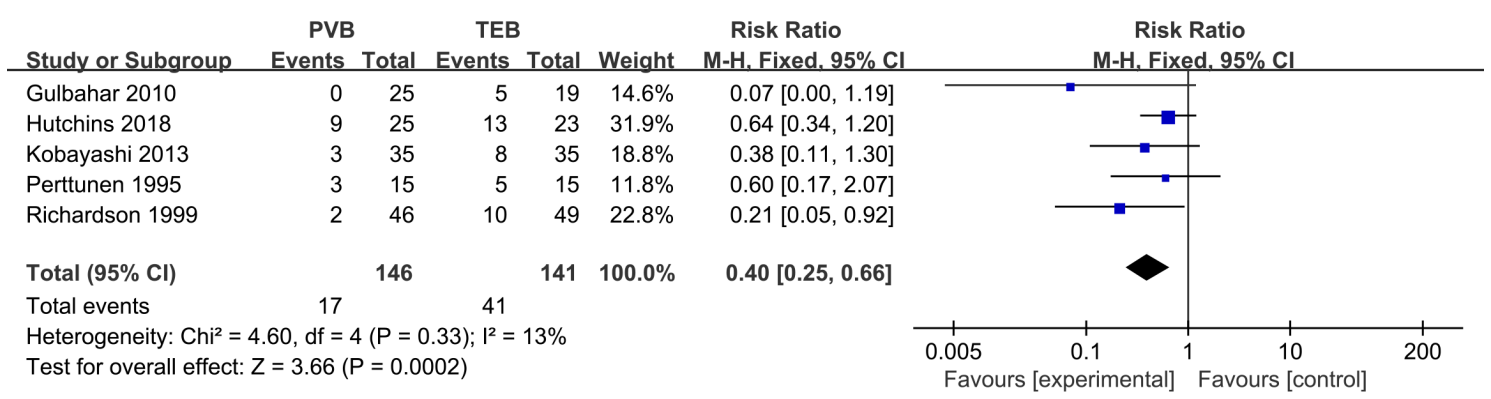

Figure 13 Forest map of fixed-effects model of nausea. PVB, paravertebral blockade; TEB, thoracic epidural blockade; df, degree of freedom; CI, confidence interval.

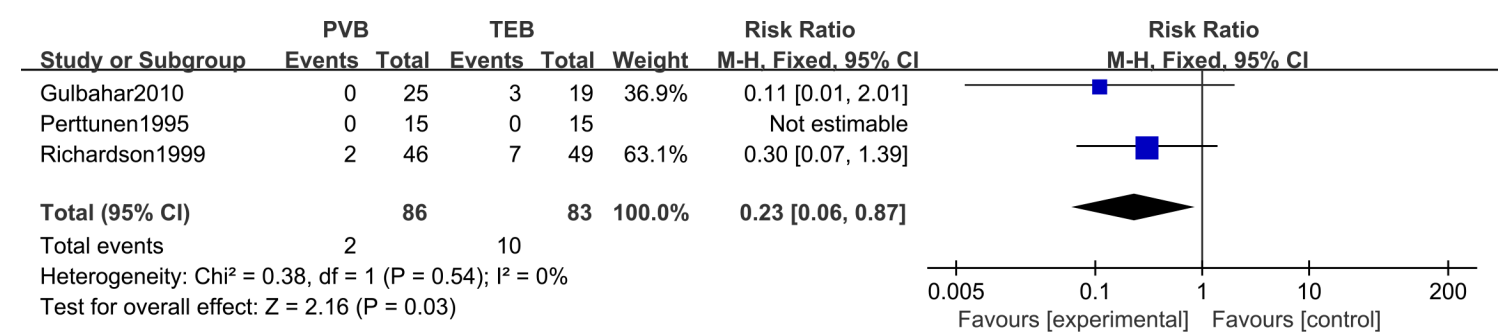

Figure 14 Forest map of fixed-effects model of vomiting. PVB, paravertebral blockade; TEB, thoracic epidural blockade; df, degree of freedom; CI, confidence interval.

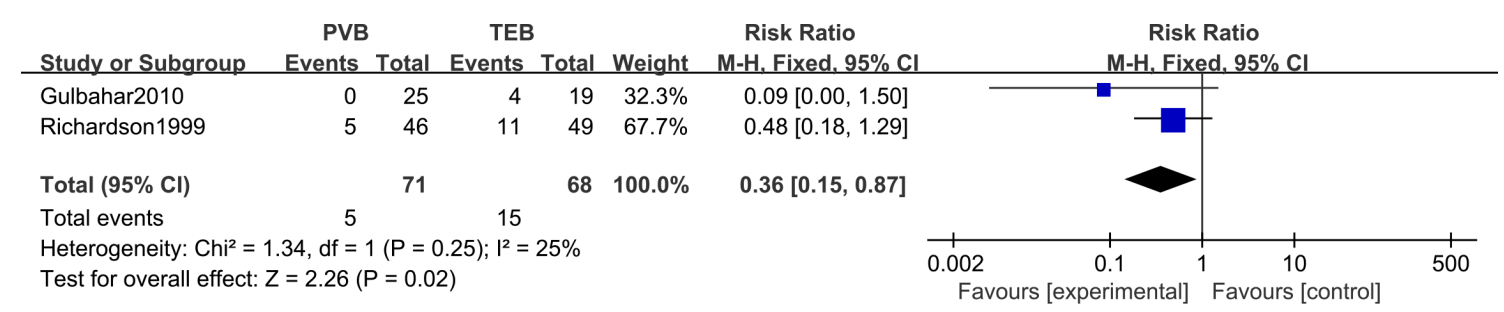

Figure 15 Forest map of fixed-effects model for incidence of urinary retention. PVB, paravertebral blockade; TEB, thoracic epidural blockade; df, degree of freedom; CI, confidence interval.

difficult during operation; therefore, the application of ultrasound greatly improves the success rate of PVB. None of the studies included in the meta-analysis in the present study reported related complications caused by puncture, but TEB puncture still has the risk of penetrating blood vessels, leading to epidural hematoma and nerve injury, which increases the risk of coagulation abnormalities and anticoagulation.

In the present study, we also analyzed the incidence of chronic pain after thoracotomy, and found that the incidence of PVB group was low (RR: 0.40, 95\% CI: 0.15$1.07, \mathrm{P}=0.07)$. However, due to the small sample size, the accuracy of the conclusion needs further verification.

\section{Conclusions}

In the present study, the related literature of PVB and epidural blockade for lung surgery was screened for systematic evaluation and meta-analysis to compare the clinical effect and safety of the 2 analgesia methods. Metaanalysis confirmed that PVB has the same analgesic effect and postoperative pulmonary function as epidural blockade in open thoracotomy lung surgery. In addition, PVB can reduce the incidence of analgesia-related complications and postoperative chronic pain. The limitation of the present study was that the sample size of the included studies was limited, and the outcome indicators of each article were not consistent, which could affect the accuracy of meta results. 


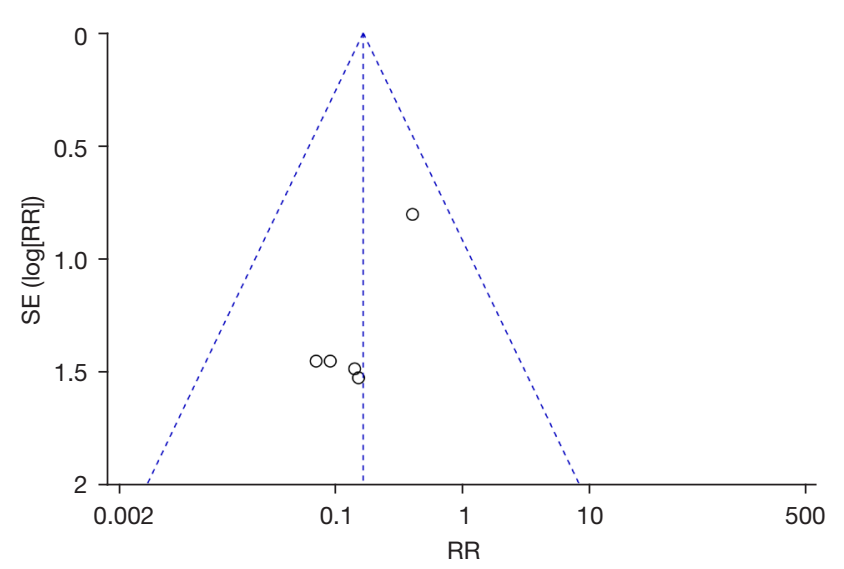

Figure 16 Funnel plot of analgesia-related complications. RR, relative risk; SE, standard error.

It will be necessary to expand the sample size to include higher quality RCTs for verification in future studies.

\section{Acknowledgments}

Funding: None.

\section{Footnote}

Reporting Checklist: The authors have completed the PRISMA reporting checklist. Available at https://jtd. amegroups.com/article/view/10.21037/jtd-22-103/rc

Conflicts of Interest: All authors have completed the ICMJE uniform disclosure form (available at https://jtd.amegroups. com/article/view/10.21037/jtd-22-103/coif). The authors have no conflicts of interest to declare.

Ethical Statement: The authors are accountable for all aspects of the work in ensuring that questions related to the accuracy or integrity of any part of the work are appropriately investigated and resolved.

Open Access Statement: This is an Open Access article distributed in accordance with the Creative Commons Attribution-NonCommercial-NoDerivs 4.0 International License (CC BY-NC-ND 4.0), which permits the noncommercial replication and distribution of the article with the strict proviso that no changes or edits are made and the original work is properly cited (including links to both the formal publication through the relevant DOI and the license).
See: https://creativecommons.org/licenses/by-nc-nd/4.0/.

\section{References}

1. Yeung JH, Gates S, Naidu BV, et al. Paravertebral block versus thoracic epidural for patients undergoing thoracotomy. Cochrane Database Syst Rev 2016;2:CD009121.

2. Blanco R, Parras T, McDonnell JG, et al. Serratus plane block: a novel ultrasound-guided thoracic wall nerve block. Anaesthesia 2013;68:1107-13.

3. El Shora HA, El Beleehy AA, Abdelwahab AA, et al. Bilateral Paravertebral Block versus Thoracic Epidural Analgesia for Pain Control Post-Cardiac Surgery: A Randomized Controlled Trial. Thorac Cardiovasc Surg 2020;68:410-6.

4. Naja MZ, Ziade MF, Lönnqvist PA. Nerve-stimulator guided paravertebral blockade vs. general anaesthesia for breast surgery: a prospective randomized trial. Eur J Anaesthesiol 2003;20:897-903.

5. Ding $X$, Jin $S, N i u X$, et al. A comparison of the analgesia efficacy and side effects of paravertebral compared with epidural blockade for thoracotomy: an updated metaanalysis. PLoS One 2014;9:e96233.

6. Kosiński S, Fryźlewicz E, Wiłkojć M, et al. Comparison of continuous epidural block and continuous paravertebral block in postoperative analgaesia after video-assisted thoracoscopic surgery lobectomy: a randomised, noninferiority trial. Anaesthesiol Intensive Ther 2016;48:280-7.

7. Hura G, Knapik P, Misiołek H, et al. Sensory blockade after thoracic paravertebral injection of ropivacaine or bupivacaine. Eur J Anaesthesiol 2006;23:658-64.

8. Burns DA, Ben-David B, Chelly JE, et al. Intercostally placed paravertebral catheterization: an alternative approach to continuous paravertebral blockade. Anesth Analg 2008;107:339-41.

9. Scarci M, Joshi A, Attia R. In patients undergoing thoracic surgery is paravertebral block as effective as epidural analgesia for pain management? Interact Cardiovasc Thorac Surg 2010;10:92-6.

10. Raveglia F, Rizzi A, Leporati A, et al. Analgesia in patients undergoing thoracotomy: epidural versus paravertebral technique. A randomized, double-blind, prospective study. J Thorac Cardiovasc Surg 2014;147:469-73.

11. Yang H, Dong Q, Liang L, et al. The comparison of ultrasound-guided thoracic paravertebral blockade and internal intercostal nerve block for non-intubated videoassisted thoracic surgery. J Thorac Dis 2019;11:3476-81. 
12. Casati A, Alessandrini P, Nuzzi M, et al. A prospective, randomized, blinded comparison between continuous thoracic paravertebral and epidural infusion of $0.2 \%$ ropivacaine after lung resection surgery. Eur J Anaesthesiol 2006;23:999-1004.

13. Grider JS, Mullet TW, Saha SP, et al. A randomized, double-blind trial comparing continuous thoracic epidural bupivacaine with and without opioid in contrast to a continuous paravertebral infusion of bupivacaine for post-thoracotomy pain. J Cardiothorac Vasc Anesth 2012;26:83-9.

14. Gulbahar G, Kocer B, Muratli SN, et al. A comparison of epidural and paravertebral catheterisation techniques in post-thoracotomy pain management. Eur J Cardiothorac Surg 2010;37:467-72.

15. Hutchins JL, Grandelis AJ, Kaizer AM, et al. Thoracic paravertebral block versus thoracic epidural analgesia for post-operative pain control in open pancreatic surgery: A randomized controlled trial. J Clin Anesth 2018;48:41-5.

16. Kaiser AM, Zollinger A, De Lorenzi D, et al. Prospective, randomized comparison of extrapleural versus epidural analgesia for postthoracotomy pain. Ann Thorac Surg 1998;66:367-72.

17. Kobayashi R, Mori S, Wakai K, et al. Paravertebral block via the surgical field versus epidural block for patients undergoing thoracotomy: a randomized clinical trial. Surg Today 2013;43:963-9.

18. Perttunen K, Nilsson E, Heinonen J, et al. Extradural, paravertebral and intercostal nerve blocks for postthoracotomy pain. Br J Anaesth 1995;75:541-7.

19. Pintaric TS, Potocnik I, Hadzic A, et al. Comparison of continuous thoracic epidural with paravertebral block on perioperative analgesia and hemodynamic stability in patients having open lung surgery. Reg Anesth Pain Med 2011;36:256-60.

20. Richardson J, Sabanathan S, Jones J, et al. A prospective, randomized comparison of preoperative and continuous balanced epidural or paravertebral bupivacaine on

Cite this article as: Ren P, Du Y, He G, Jiang D. Efficacy and safety of general anesthesia combined with paravertebral blockade on postoperative recovery in patients undergoing pulmonary surgery: a systematic review and meta-analysis. J Thorac Dis 2022;14(2):431-442. doi: 10.21037/jtd-22-103 post-thoracotomy pain, pulmonary function and stress responses. Br J Anaesth 1999;83:387-92.

21. Pace MM, Sharma B, Anderson-Dam J, et al. UltrasoundGuided Thoracic Paravertebral Blockade: A Retrospective Study of the Incidence of Complications. Anesth Analg 2016;122:1186-91.

22. Re M, Blanco-Murcia J, Villaescusa A, et al. Comparison of paravertebral blockade techniques with and without ultrasound guidance in calves. Am J Vet Res 2016;77:1187-93.

23. Neuburger PJ, Ngai JY, Chacon MM, et al. A Prospective Randomized Study of Paravertebral Blockade in Patients Undergoing Robotic Mitral Valve Repair. J Cardiothorac Vasc Anesth 2015;29:930-6.

24. Koyyalamudi VB, Arulkumar S, Yost BR, et al. Supraclavicular and paravertebral blocks: Are we underutilizing these regional techniques in perioperative analgesia? Best Pract Res Clin Anaesthesiol 2014;28:127-38.

25. Gómez Rivas J. Last minute cancelations in elective surgery are inevitable. Can we reduce them? An invited commentary on "reduction of last-minute cancellations in elective urology surgery: A quality improvement study". Int J Surg 2020;74:109-10.

26. Neuburger PJ, Chacon MM, Luria BJ, et al. Does paravertebral blockade facilitate immediate extubation after totally endoscopic robotic mitral valve repair surgery? Innovations (Phila) 2015;10:96-100.

27. Kelly ME, Mc Nicholas D, Killen J, et al. Thoracic Casati paravertebral blockade in breast surgery: Is pneumothorax an appreciable concern? A review of over 1000 cases. Breast J 2018;24:23-7.

28. Visoiu M, Cassara A, Yang CI. Bilateral Paravertebral Blockade (T7-10) Versus Incisional Local Anesthetic Administration for Pediatric Laparoscopic Cholecystectomy: A Prospective, Randomized Clinical Study. Anesth Analg 2015;120:1106-13.

(English Language Editor: R. Scott) 